\title{
A Common Research Hypothesis for Crohn Disease, Ulcerative Colitis, Psoriasis and Acquired Vitiligo
}

\author{
Tapasyapreeti Mukhopadhyay ${ }^{1}$, Amritapreeti Mukhopadhyay ${ }^{2}$ and Asok Kumar Mukhopadhyay ${ }^{3}$ \\ ${ }^{1}$ Department of Laboratory Medicine, All India Institute of Medical Sciences, New Delhi, India \\ ${ }^{2}$ Kasturba Medical College, Mangalore, India \\ ${ }^{3}$ Department of Pathology, North DMC Medical College and HRH, Delhi, India
}

Submission: May 07, 2019; Published: May 28, 2019

*Corresponding author: Asok Kumar Mukhopadhyay, Department of Pathology, North DMC Medical College and HRH, Delhi, India

\begin{abstract}
Two polar views that a disease has its genesis in the mind and the disease has its source in the dysfunction of cell have been tried to be bridged by formulating a common research hypothesis for Crohn disease, vitiligo, ulcerative colitis and psoriasis. This has been done on the basis of existing facts/evidences which, although are not complete by any sense, have been aligned and integrated horizontally and vertically. Since all four diseases are autoimmune in nature related to microbiota, have a genetic predisposition, and the tissue damage occurs by a common hypersensitivity reaction, the proposed conceptual framework is stimulating for a massive poly-disciplinary research on Psycho-neuroendocrine-immune axis and on the genetic basis of the diseases. The perspective has far reaching implications extending from environmental sanitation and psychic hygiene to cell biology and hygiene hypothesis of disease.
\end{abstract}

Keywords: Research Hypothesis; Personality traits; Microbiota; Autoimmune response; Etiopathogenesis of IBD; Psoriasis; Vitiligo

\section{Introduction}

Behaviour, thoughts and feelings of the individual subject specify his/her personality trait. The role of personality characteristics in developing psychosomatic illness, although, is not a new topic $[1,2]$, no concrete hypothesis, however, is found to exist for any such disease. In pathogenesis of disease of autoimmune origin, the psycho immuno-axis, as known today, is incomplete or even non-existent. Although the mind of the sufferer is presumed to have an influence on hypersensitive tissue damage reaction, the probable linkage with its weaponry at molecular level, we mean cytokines, is missing.

That all diseases involve alteration of functions of normal cells, or, all pathology ultimately is cellular pathology is an eminent view of the cell theorist Rudolf Virchow [3]. Another view also prevails; a disease has its origin in the Psyche (Mind) especially when the systems psyche gets disconnected from the Whole. This view is held by most psychologists and spiritualists but lacks evidence. Could this latter view be supported by any evidence from the pathological basis of diseases? This question motivated us to pick up four diseases, Crohn disease, ulcerative colitis, psoriasis and acquired vitiligo and to look for a research framework in establishing their common origin embracing the two views on mechanism in origin of disease.

\section{Existing facts}

Inflammatory Bowel Disease (IBD) i.e. Crohn Disease (CD) and Ulcerative Colitis (UC), and psoriasis and acquired vitiligo of skin are diseases having psychosomatic connection in etiology and common immune-hypersensitivity response mechanism in pathogenesis. The former two [4] affect the intestine and present with chronic diarrhoea and malabsorption syndrome. The latter two are well-known skin disease; psoriasis [5] is a patchy chronic inflammatory dermatosis with bumpy red patches covered with white scales that persists for years, and acquired vitiligo [6] results from melanocyte destruction in the skin resulting in depigmented white maculo-papular patches localised or spread over the region or distributed segmentally.

What these four diseases have in common are as follows:

i. All four diseases can occur at any age and are on the surface of the system; IBD affects the mucosal surface of GI system, while psoriasis and vitiligo affect the dermatological system.

ii. The diseases are chronic, persists for years, almost life-long, although non-infective but characterized by sterile inflammation. 
iii. All four are of autoimmune origin where autoimmunity is not against body tissue or any known infective organism. In development of this autoimmunity, physiological microbiota of the respective surface is actively involved [7], the mechanism, although, remains unclear. Hygiene hypothesis has an established role in epidemiology of IBD. Emerging data also speak of the same for the two dermatoses.

iv. Immunological response is of delayed type, i.e. type IV hypersensitivity reaction dominated by T-Helper lymphocyte response. TH1 and TH17 cell gather there in the site of all four diseases. The role of togetherness of TH1 and TH 17 cell is well established in organ specific autoimmune disease [8] where the balance between function of TH17 cells and Treg cell is important [9]. The tissue damage is caused by hypersensitivity reaction by the respective cytokines of TH1 and TH17 cells [10].

v. All four diseases are characterised by spontaneous remission and exacerbation, related to the degree and kind of stress in the mind of the patient. There is lack of literature to reach any conclusion on if there is any specific polarity of personality traits which could have influences in the development of these diseases.

Accordingly, from two polar personality traits, there is bipolar $\mathrm{T}$ regulatory cell response. $\mathrm{T}$ helper cell response is also bifurcated as predominant TH1 response leading to granuloma formation as in Crohn Disease and in some cases of Vitiligo, and predominant TH17 response with development of ulcerative lesion with neutrophilic exudates as in ulcerative colitis and psoriasis.

\section{Building up Vertical Integration}

The question why some patients develop ulcerative colitis (and psoriasis) and others develop Crohn disease (and vitiligo, or "cutaneous metastasis" of Crohn disease) prompted us to relate the diseases with bipolar personality trait. Specific personality trait determines the life-style of the patients and susceptibility to environmental stressors. The subjects with low self-esteem, depressive trait, alexithymia (deficits of emotional awareness and inability to give a name to emotional states) are prone to develop granulomatous response in inflammation as we see in tuberculosis, Crohn disease (and in some cases of vitiligo with papules containing granuloma). On the other side, subjects with restlessness, impulsive drives, emotionally charged manic personality trait, if ever develop any organ-specific autoimmune disease involving the body surface, develop a 'juicy' immune inflammation in the intestine, as found in ulcerative colitis and in the skin as found in psoriasis. There are papers relating personality traits with IBD [11], psoriasis [12] and psychiatric morbidity in vitiligo [13].

Since in some subjects, the personality trait is not absolutely stable but swings between two poles, we often find a mix of UC and CD (often diagnosed as indeterminate type of IBD) in the intestine and coexistence of vitiligo and psoriasis in the skin of patient. However, vitiligo has been reported to be associated with other autoimmune disease like systemic lupus erythematosus, Addison's disease, autoimmune thyroid disease [14,15].

The vertical link between personality and immune function is a major frontier of recent research. The relation between positivity-negativity of thoughts and immune regulation [16], immune response in conscientious versus carefree individuals, immune dysfunction in rigid versus resilience personality trait are major thrust area in establishing this vertical link [17]. This link is conspicuous by the paucity of evidence in genesis of these four autoimmune diseases, which are concern of this paper.

\section{Horizontal Integration and Alignment of the Systems}

Alignment and integration of the psychological and immunological system is vertical in the proposed hypothesis. Alignment of psoriasis and vitiligo is part of horizontal integration within the dermatological system. Similar is the alignment of Crohn Disease and ulcerative colitis in gastrointestinal system. Corresponding microbiota is somehow involved in the auto immune process in all four diseases. Not only there exists a vertical axis between gut microbiota and brain dynamics [18], also there is an inter-systems axis between gut microbiota and skin microbiota [19]. The skin microbiota is involved in developing many skin diseases [20], including psoriasis [21,22] and vitiligo [23]. This is now a text book knowledge that the intestinal microbiota is involved in pathogenesis of UC and CD.

Therefore, we have alignment of the pathogenetic processes both within the systems and between the systems of skin and GI system. Inter-systems association is exemplified by association of Psoriasis with IBD [24] and association of Vitiligo and Crohn disease in children [25] and aggravation of vitiligo following major surgery for Crohn disease [26]. In the same system, case reports exist where both the paired disease can coexist. Coexistence of UC and CD has been reported by Chen et al. [27]. There is case report of patients having concurrent vitiligo and psoriasis [28,29].

We still do not know where all such evidence lead us to? Therefore, there is a scope for formulation of an overarching hypothesis which could stimulate more research of poly disciplinary nature!

\section{The Proposed Hypothesis}

Two specific traits of personality, Vitiligo-CD personality trait and Psoriasis-UC personality trait, are prone to develop two types of immune dysregulation involving Treg cell and normal microbiota of intestine and skin. This leads to TH cell response with a bifurcation into predominant TH1 response with granuloma formation (as in Crohn disease and in vitiligo), and predominant TH17 response with ulceration and exudates (as in UC and psoriasis). Since physiological microbiota of intestine 


\section{Psychology and Behavioral Science International Journal}

and skin work through an intersystem communicative axis, on the basis of which of TH17 or TH1 responses predominating UC patients is often associated with psoriasis and CD patients often with vitiligo (Figure 1).

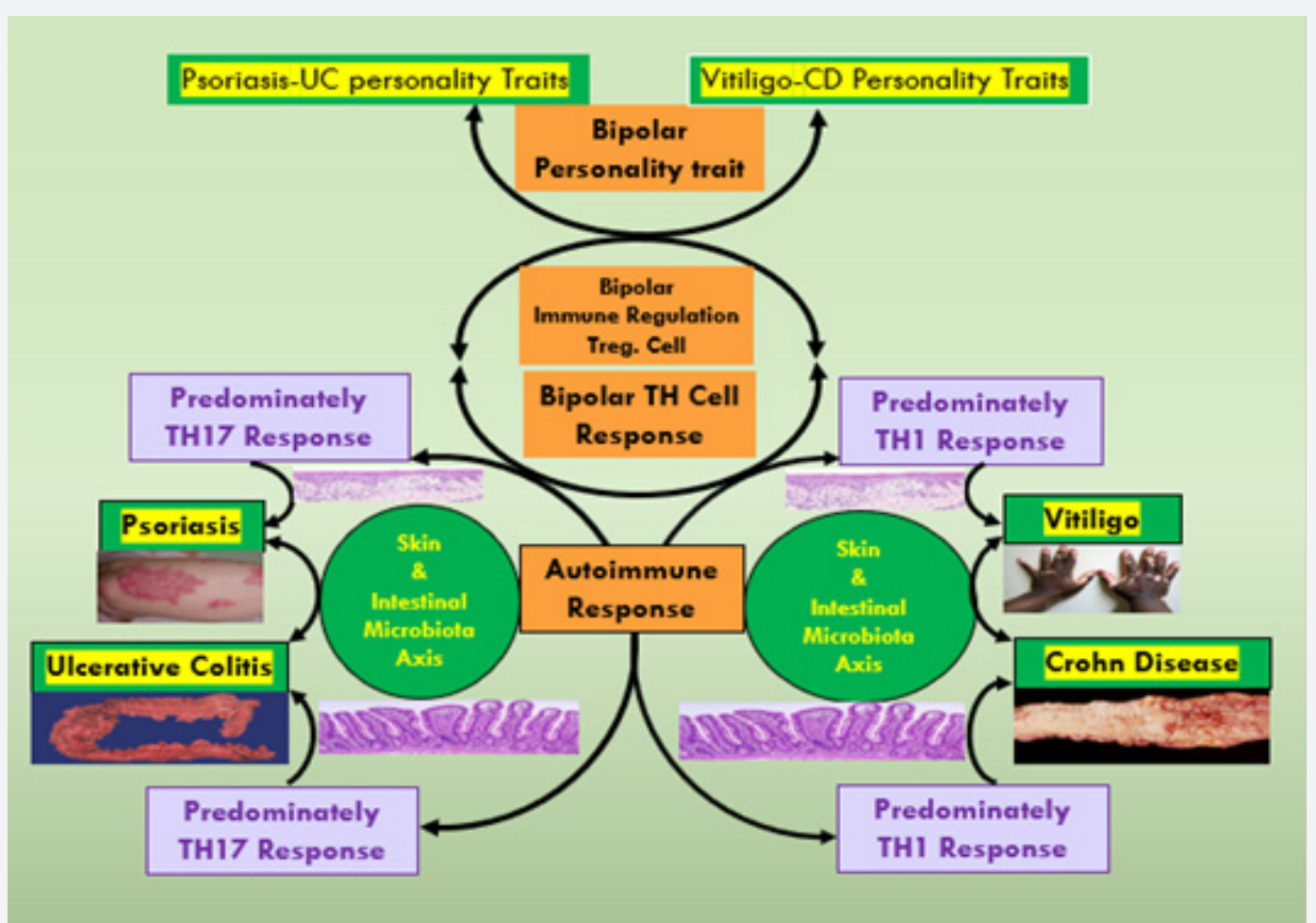

Figure 1: Ulcerative colitis, Crohn disease, Psoriasis and Vitiligo have been framed on a quadrangle of common research hypothesis with two specific types of personality traits, two types of immune regulation (Treg cells) involving two types of T helper (TH) cell response being together but predominating as either TH1 or TH17 way resulting in morphological expression as granuloma (with strictly TH1 related cytokines) or ulcer (TH17 related cytokine "soup"). Either or distinction is not very rigid along the whole vertical axis from the Top to the Bottom because of not-so-rigidity of personality traits. Autoimmune response against normal microbiota remains at the centre of the framework, where the autoimmunity is built up in the respective surface epithelium. The framework of the hypothesis makes the gaps conspicuous and stimulates for further research.

\section{Major and Minor Gaps in the Hypothesis}

The hypothesis made is tentative and is a working hypothesis to start with. There are many gaps in vertical as well as horizontal alignments. There is a need for a massive workout on the following lines, which form the major gaps in the hypothesis.

i. In spite of having some good published papers connecting personality traits with IBD, psoriasis, and vitiligo as mentioned earlier, more research is needed on multidimensional and multi-layered personality trait analysis of patients suffering from IBD, psoriasis and vitiligo. The role of "little" versus "big" traits may also be investigated. We are yet to develop specifications for Vitiligo-CD personality trait and Psoriasis-UC personality trait.

ii. To collect evidence in support of connection between the said binary personality trait and binary immune regulation.

iii. To gather evidence in support of binary immune regulation (Treg cell) and binary TH1 and TH17 response. iv. How and when normal microbiota of intestine or of skin get involved in the process of autoimmunity?

v. How mental stress and anxiety trait relate to "spontaneous" partial remission and exacerbation of the disease?

There are also minor gaps which deserve explanations.

i. Cutaneous granulomatosis in Crohn disease has been reported [30,31]. There is also case report of colocalization of vitiligo and elastolytic granuloma [32]. There is case report of association of vitiligo with cutaneous sarcoidosis showing granuloma [33]. The question remains why granulomatous reaction is not usual or not so common in vitiligo? And, is there any relation between segmental occurrence of $\mathrm{CD}$ and segmental Vitiligo?

ii. Association of Psoriasis with IBD is well known, as stated. The question is, are psoriasis and IBD two sides of the same coin [34]? 
iii. What is the exact role of skin microbiota in developing psoriasis and vitiligo?

Finally, HLA association, and chromosomal and genetic analysis of all four diseases appears rewarding since all four of them have defined genetic predisposition. We are to decipher what is the common denominator for them. Efforts are there to redefine IBDs using Genome-scale molecular typing [35]. Many of us are not sure whether such extensive genetic analysis will have any benefits for the patients! However, gap analysis and further research on common gene denominators for these four diseases is expected to open up new doors of memes-genes interaction in psychosomatic connection [36].

\section{Discussion}

The pathologists do not work professionally with extrasensory perception [37]. Neither the clinicians practice with! The profession of medicine is evidence-based. Hypothesis generation is necessary because of existence of gaps which do not allow the process of overarching. This paper has been generated for an impending massive work to establish the psycho neuro immune and psycho neuro endocrine immune axis in pathogenesis of cellular origin of disease. The hypothesis awaits testing based on this multidisciplinary research project, which encompasses horizontally gastroenterology (medicine as well as surgery) and dermatology, and integrating both systems vertically with pathology (biopsy, immune histochemistry) and laboratory medicine (flow cytometry, molecular biology, hormones and neurotransmitter analysis), immunology (flow cytometry, immune cytochemistry etc.) and psychology/ psychiatry (multidimensional multi-layered personality trait analysis). Besides, this hypothesis could be a part of the larger paradigm of the origin of disease in mind prior to the origin of disease in cell under the umbrella of the Worldview that disease originates because of disconnection from the Whole!

In the formulation stated above and shown in figure 1, there is no 'neuro' component. However, one could be serious about how Multiple sclerosis, an autoimmune disease of nervous system, mediated by similar TH1 and TH17 response, could be accommodated in the scheme. There is so far no evidence of normal physiological micro flora existing in the nervous system although there are literatures on gut micro flora influencing the brain dynamics [18]. Next question regarding the nervous system specific auto immune disease is, what could be the other disease for pairing with multiple sclerosis? Could it be a humanequivalent of experimental autoimmune encephalitis in animals, where TH1 cells are seen to facilitate entry of TH17 in the site [38]?

The traditional vertical integration between psychology and immunology is being done through nervous system and endocrine system and on this basis we talk of psychoneuroimmune axis or psycho neuro endocrine immune axis. However, if we consider that every living entity including every cell of the body possesses the organ psyche, then the change or alteration in the systems psyche could directly affect the cellular psyche! This could be an example of instantaneous communication at distance in psychobiology. In this mechanism, molecular links also have been proposed $[39,40]$. Two extreme theories i.e., origin of disease in mind and origin of disease within cell could be reconciled by advancing research on the propositions made in these papers.

\section{Concluding Remarks and Perspective}

In this research hypothesis we cover four diseases involving the skin surface and gastro intestinal mucosa. None of the four diseases has cure. Anti-inflammatory drugs have failed. Surgery of IBD leads to no spectacular success. Stem cell transplantation is on trial. All such developments and failures lead us back to the bottom of the disease, the microbiota. Intestinal microbiota is related to health and disease of the skin through a complex communication network between the immune system, endocrine system, metabolic system, and nervous system. Since skin microbiota and gut microbiota have an axis of interaction, there opens therapeutic doors of ameliorating skin lesions with prebiotics and probiotics. Faecal transplantation in IBD in order to alter intestinal microbiota has been on trial.

Could the hypothesis be extended to accommodate the epithelium and microbiota of respiratory and urogenital systems? Probably not! GIT is open both sides and communicates with skin both sides. Respiratory and urogenital systems are not open both sides. However, Idiopathic pulmonary fibrosis has unflinching evidence of autoimmune origin. The evidence has been accumulating in experimental animals that perhaps the pulmonary emphysema could have autoimmune origin [41]. Nothing is known yet about any such disease involving the urogenital epithelium!

In perspective, therefore it could be said that this small quadrangular nidus created in this hypothesis has the potential to accommodate a large number of psychosomatic diseases, which are organ specific autoimmune in nature. Genetic and molecular analysis of these diseases could also open up new widows for genetic basis of such disease. The hypothesis raises profound questions like, could the state of psyche in general and the state of mind in particular alter physiological microbiota of skin, intestine, respiratory and genital systems? What is the relation between psychic hygiene, environmental hygiene and the hygiene hypothesis in pathogenesis of these autoimmune diseases? To establish this relation, we require evidence. Mere gut feelings, extrasensory perception or intuition will be of little help!

\section{Declaration}

No funding agency funded for production of this paper. Authors declare that there is no conflict of interest amongst them.

\section{Acknowledgement}

The medical graduation (MBBS) students of fourth semester in North DMC Medical College and Hindu Rao Hospital, 
Delhi were the stimulant to write this paper, following Dr. A. K. Mukhopadhyay finished his lecture series for them on Hypersensitivity reactions, Autoimmunity, Inflammatory Bowel disease and related discussion on psoriasis and vitiligo. Dr. Rajbala Yadav, Professor of Pathology also contributed with clinical examples from her life-long experience in pathology and clinical medicine. Dr. Ruchi Rathore, Asst. Professor in Pathology, also participated during the discussion. Dr. Jay Relan, senior resident in Pediatric cardiology at AIIMS, Delhi helped in editing. Anindya Sundar Mukhopadhyay helped to improve the design of the figure. Authors acknowledge their contribution. We also thank Mrs. Pooja Taneja for secretarial assistance.

\section{References}

1. Kaplan H (1985) Psychological factors affecting physical conditions (psychosomatic disorders). Comprehensive textbook of psychiatry IV 4:1106-1113.

2. Lyketsos CG (1987) Personality Characteristics in Psychosomatic Illness. Psychosomatic Medicine: 65-72.

3. Schultz M (2008) Rudolf Virchow. Emerging infectious diseases 14 (9): 1480-1481.

4. Colombel JF and Mahadevan U (2017) Inflammatory Bowel Disease 2017: Innovations and Changing Paradigms. Gastroenterology 152(2): 309-312.

5. Marzano AV, Derlino F, Berti EF (2018) Pathogenesis of Psoriasis: Focus on Autoinflammation. Dermatopathology 5: 14-15.

6. Arora AK, Kumaron MS (2017) Pathogenesis of Vitiligo: An update. Pigment International 4(2): 65-77.

7. Gianchecchi E, Fierabracci A (2019) Recent Advances on Microbiota Involvement in the Pathogenesis of Autoimmunity. Int J Mol Sci 20: 283.

8. Dardalhon V, Kom T, Kuchroo VK, Anderson AC (2008) Role of Th1 and Th17 cells in organ-specific autoimmunity. J Autoimmun 31(3): 252256.

9. Lee GR (2018) The Balance of Th 17 versus Treg cells in Autoimmunity. International Journal of Molecular Sciences 19 (3): 730.

10. Koloski NA, Bret L, Radford-Smith G (2008) Hygiene hypothesis in inflammatory bowel disease: a critical review of the literature. World J Gastroenterol 14(2): 165.

11. Barbera DL, Bonanno B, Rumeo MV, Alabastro V, Frenda M, et al (2017) Alexithymia and personality traits of patients with inflammatory bowel disease. Sci Rep 7: 41786.

12. Lim DS, Bewley A, Oon HH (2018) Psychological Profile of Patients with Psoriasis. Ann Acad Med Singapore 47(12): 516-522.

13. Sarkar S, Sarkar T, Sarkar A, Das S (2018) Vitiligo and Psychiatric Morbidity: A Profile from a Vitiligo Clinic of a Rural-based Tertiary Care Center of Eastern India. Indian J Dermatol 63(4): 281-284.

14. Nejad SB, Qadim HH, Nazeman L, Fadaii R, Goldust M (2013) Frequency of autoimmune diseases in those suffering from vitiligo in comparison with normal population. Pak J Biol Sci 16 (12): 570-574.

15. Spritz RA (2010) Shared genetic relationships underlying generalized vitiligo and autoimmune thyroid disease. Thyroid 20(7): 745-754.

16. Segerstrom SC, Sephton SE (2010) Optimistic expectancies and cellmediated immunity: The role of positive affect. Psychological Science 21: 448-455.
17. Cohen S, Janicki-Deverts D, Crittenden CN, Sneed RS (2012) Personality and Human Immunity. In: Segerstrom, S. (Ed.). The Oxford Handbook of Psychoneuroimmunology. Oxford University Press, United Kingdom, pp. 146-169.

18. Ma Q Xing C, Long W, Wang HY, Liu Q (2019) Impact of microbiota on central nervous system and neurological diseases: the gut-brain axis. Journal of Neuroinflammation 16: 53.

19. Vaughn AR, Notay M, Clark AK, Sivamani RK (2017) Skin-gut axis: The relationship between intestinal bacteria and skin health. World J Dermatol 6(4): 52-58.

20. Abdallah F, Mijouin L, Pichon C (2017) Skin Immune Landscape: Inside and Outside the Organism. Mediators of Inflammation: 5095293.

21. Benhadou F, Mintoff D, Schnebert B, Thio HK (2018) Psoriasis and Microbiota: A Systematic Review. Diseases 6(2): 47.

22. Chang HW, Yan D, Singh R, Liu J, Lu X, et al. (2018) Alteration of the cutaneous microbiome in psoriasis and potential role in Th17 polarization. Microbiome 6: 154 .

23. Ganju P, Nagpal S, Mohammed MH, Kumar N, Pandey R, et al.(2016) Microbial community profiling shows dysbiosis in the lesional skin of Vitiligo subjects. Scientific Reports 6: 18761.

24. Fu Y, Lee CH, Chi CC (2018) Association of Psoriasis with Inflammatory Bowel Disease: A Systematic Review and Meta-analysis. JAMA Dermatol 154(12): 1417-1423.

25. Pashankar D, Prendiville J, Israel DM (1999) Vitiligo and Crohn's disease in children. J Pediatr Gastroenterol Nutr 28 (2): 227-229.

26. Triantafillidis JK (2013) Vitiligo aggravated after major surgery for Crohn's disease: a consequence of severe surgical stress? Ann Gastroenterol 26 (3): 278-279.

27. Chen GI, Saibil F, Morava-Protzner I (2002) Two for one: coexisting ulcerative colitis and Crohn's disease. Can J Gastroenterol.16(1): 29-34.

28. Chakraborty D, Haneef ND, Razvi F, Praveen Kumar BY, Fatima, N et al. (2017) A case report showing coexistence of two autoimmune diseases-psoriasis and vitiligo. J Med Allied Sci 7(2): 114-117.

29. Park JM, Kim HJ, Byung Gi Bae BG, Yoon Kee Park YK (2009) A Case of Concurrent Vitiligo and Psoriasis. Ann Dermatol 21(3): 330-333.

30. Levine N, Bangert J (1982) Cutaneous Granulomatosis in Crohn's Disease. Arch Dermatol 118(12): 1006-1009.

31. McPoland PR, Moss RL (1988) Cutaneous Crohn's disease and progressive vitiligo. J Am Acad Dermatol 19: 421-425.

32. de Paz NM, Rodríguez-Martín M, Bustínduy MG, Martín-Herrera A, Noda-Cabrera A (2010) Strict Anatomical Colocalization of Vitiligo and Elastolytic Granulomas. Case Rep Dermatol 2(1): 13-17.

33. Lalosevic J, Skiljevic D (2014) Cutaneous Sarcoidosis Associated with Vitiligo and Autoimmune Thyroiditis - a Case Report. Serbian Journal of Dermatology and Venereology 6 (3): 138-142.

34. Fiorino G, Omodei PD (2015) Psoriasis and Inflammatory Bowel Disease: Two Sides of the Same Coin? Journal of Crohn's and Colitis 9 (9): 697-698.

35. Furey TS, Sethupathy P, Sheikh SZ (2019) Redefining the IBDs using genome-scale molecular phenotyping. Nat Rev Gastroenterol Hepatol 16 (5): 296-311.

36. Mukhopadhyay AK (2010) The Self and its Memes and Genes: Genes, Memes, Self (Brain), Information and Consciousness. In: History of Science and Philosophy of Science. A Historical Perspective of the Evolution of Ideas in Science. Project History of Indian Science, Philosophy and Culture (PHISPC) Vol. XI, Part VI, Chapter 21. Ed. Pradip Sengupta. Pub. Pearson, New Delhi, India: pp. 481-558. 
37. Bull AD, Cross SS, James DS, Silcocks PB (1991) Do pathologists have extrasensory perception? BMJ 303 (6817): 1604-1605.

38. O'Connor RA, Prendergast CT, Sabatos CA, Lau CW, Leech MD, et al. (2008) Cutting edge: Th1 cells facilitate the entry of Th17 cells to the central nervous system during experimental autoimmune encephalomyelitis. J Immunol 181(6): 3750-3754.

39. Mukhopadhyay AK (2015) Systems Cell: A Testable Model for Systems Holism. International Archives of Medicine 8 (104): 1-10.
40. Mukhopadhyay AK (2016) Systems Psyche: It's Structure, Operation and possible Molecular links Psychol Behav Sci Int J 1: 555565.

41. Kheradmand F, Shan M, Chuang Xu C, Corry DB (2012) Autoimmunity in chronic obstructive pulmonary disease: clinical and experimental evidence. Expert Rev Clin Immunol 8(3): 285-292.

\section{Your next submission with Juniper Publishers will reach you the below assets}

- Quality Editorial service

- Swift Peer Review

- Reprints availability

- E-prints Service

- Manuscript Podcast for convenient understanding

- Global attainment for your research

- Manuscript accessibility in different formats

( Pdf, E-pub, Full Text, Audio)

- Unceasing customer service

Track the below URL for one-step submission https://juniperpublishers.com/online-submission.php 